\title{
Fluoride varnish should be part of caries prevention programmes
}

\author{
In pre-school children is fluoride varnish application in addition to \\ counselling more effective than counselling alone in preventing early \\ childhood caries?
}

Weintraub JA, Ramos-Gomez F, Jue B et al.

Fluoride varnish efficacy in preventing early childhood caries.

J Dent Res. 2006; 85:172-176

Design Randomised controlled trial in two community health centres. Intervention All families received counselling, and children were randomised to the following groups: no fluoride varnish, fluoride varnish once a year, or fluoride varnish twice a year. Due to an unexpected protocol variation children unintentionally received a placebo varnish instead of the active product during a 10-month period. Consequentially only one child received all four planned active applications.

Outcome measure The primary outcome measure was dental caries assessed by one dentist masked to treatment group. Two-year follow-up was planned unless caries were detected at the 1-year follow-up examination, in which case children were considered treatment failures and referred for dental care.

Results A total of 376 children were randomised with a mean age of 1.8 years of whom $71 \%$ were examined at year 1 and $67 \%$ at year 2 . Intent-to-treat analyses showed a fluoride varnish protective effect in caries incidence, $P<0.01$. Analysing the number of actual, active fluoride varnish applications received resulted in a dose-response effect, $\mathrm{P}<$ 0.01 . Caries incidence was higher for counselling only vs 'counselling + fluoride varnish assigned once/year' (odds ratio, 2.20; $95 \%$ confidence interval 1.19-4.08) and 'twice/year' (odds ratio, 3.77; 95\% confidence interval 1.88-7.58). No related adverse events were reported.

Conclusions Fluoride varnish and parental counselling should be recommended as part of caries prevention programmes targeting infants and toddlers.
Address for correspondence: Dr J Weintraub, Center to Address Disparities in Children's Oral Health and Comprehensive Oral Health Research Center of Discovery, University of California, San Francisco School of Dentistry, 3333 California Street, Suite 495, San Francisco, CA 94143-1361, USA.

\section{Commentary}

This trial, conducted in a community setting with low income preschool children, adds to the evidence on the effectiveness of fluoride varnish in preventing caries. It is an area that has undergone extensive study and systematic review. However, the majority of the studies have been conducted on school age children. The Cochrane review ${ }^{1}$ included only three studies on pre-school children, with a pooled estimate for the $\mathrm{d}(\mathrm{m} / \mathrm{e})$ fs prevented fraction $(\mathrm{PF})$ of $33 \%$ (95\% confidence interval, 19 to $48 \%$; $<<0.0001$ ), which they suggested indicated a substantial benefit of fluoride varnish in the deciduous dentition. The $2001 \mathrm{NIH}$ consensus conference ${ }^{2}$ was more circumspect, stating, "The evidence for the benefit of applying fluoride varnish to permanent teeth is generally positive. In contrast, the evidence for effectiveness of fluoride varnish applied to primary teeth is incomplete and inconsistent".

The trial was well conducted and sufficiently powered to show differences between the groups despite the protocol violation caused by the provision of placebo varnish which is clearly explained. Those children randomised to the annual application of fluoride varnish had a PF of $58 \%$ and those who were randomised to a biannual application a PF of $61 \%$. When analysed according to the number of active fluoride applications received those with one application had a PF of $53 \%$, those with two applications a PF of 58\% and those with three of four had a PF of $93 \%$.

The PF achieved in this study are much higher than those found in the Cochrane review PF and clearly support the efficacy of this intervention. While the figures for PF are much better than the Cochrane review, the numbers needed to treat (NNT) shown in Table1 are much higher because the trial is looking at the caries free child as an outcome rather than a reduction in caries.

Table 1. Numbers need to treat to have one extra child caries free at two years

\begin{tabular}{lccc}
\hline & Counselling & $\begin{array}{l}\text { Counselling + } \\
\text { fluoride varnish } \\
\text { assigned once/ } \\
\text { year }\end{array}$ & $\begin{array}{l}\text { Counselling } \\
\text { fluoride } \\
\text { varnish } \\
\text { assigned } \\
\text { twice/year }\end{array}$ \\
\hline Number & 126 & 124 & 126 \\
\hline $\begin{array}{l}\text { Number with no } \\
\text { caries at 2 years }\end{array}$ & $48(38.1 \%)$ & $59(47.6 \%)$ & $67(53.2 \%)$ \\
\hline $\begin{array}{l}\text { Numbers needed } \\
\text { to treat (95\% Cl) }\end{array}$ & & $11(5-37)$ & $7(4-34)$ \\
\hline
\end{tabular}

$\mathrm{Cl}$, Confidence interval

The benefit of even a single application of the varnish was shown, as well as a dose-response gradient, a point that is worth nothing in this group of patients, who may well be infrequent attenders. 
The fluoride application was well accepted by the children even under the age of 1 year. While in this study the fluoride application was conducted by a dentist, it is a non-invasive, relatively straightforward procedure that could be performed by other members of the dental team. The use of other members of the team or even other appropriate health centre staff could be an effective way of delivering this effective preventive measure in under-served areas.

This trial was included in the evidence reviewed by the American Dental Association for their evidence-based recommendations for professional topical fluoride applications ${ }^{3}$ (see page 62) who recommended 6-monthly applications of fluoride varnish for all except those at low risk of dental caries in line with other evidence-based guidelines. ${ }^{4,5}$ This is a recommendation that I would wholeheartedly support.

\section{Practice point}

Topical fluoride varnish should be applied to the dentition at least twice yearly for pre-school children assessed at increased risk of dental caries.

\section{Derek Richards}

Director, Centre for Evidence-based Dentistry, Oxford, UK.

1. Marinho VCC, Higgins JPT, Logan S, Sheiham A. Fluoride varnishes for preventing dental caries in children and adolescents. Cochrane Database of Systematic Reviews 2002, issue 1.

2. Anon. Diagnosis and management of dental caries throughout life. National Institutes of Health Consensus Development Conference statement, March 26-28, 2001. J Dent Educ. $2001 ; 65: 1162-1168$.

3. Anon. Professionally applied topical fluoride: Evidence-based clinical recommendations. J Am Dent Assoc 2006;137:1151-1159.

4. Anon. Preventing dental caries in children at high caries risk targeted prevention of dental caries in the permanent teeth of 6-16 year olds presenting for dental care. Edinburgh: Scottish Intercollegiate Guideline Network (SIGN). 2000; SIGN No 47. ISBN 1899893326.

5. Anon. Prevention and management of dental decay in the pre-school child. Edinburgh: Scottish Intercollegiate Guideline Network (SIGN). 2005; SIGN No 83. ISBN 1899893 44X.

Evidence-Based Dentistry (2006) 7, 65-66

doi:10.1038/sj.ebd.6400123x 